\title{
Publisher's Erratum to: The Herschel PACS photometer calibration
}

\section{A time dependent flux calibration for the PACS chopped point-source photometry AOT mode}

\section{Markus Nielbock • Thomas Müller • Ulrich Klaas • Bruno Altieri • Zoltán Balog • Nicolas Billot • Hendrik Linz • Koryo Okumura • Miguel Sánchez-Portal • Marc Sauvage}

Published online: 5 July 2014

(c) Springer Science+Business Media Dordrecht 2014

Erratum to: Exp. Astron. 36, 631-660 (2013)

DOI 10.1007/s10686-013-9348-z

Due to an oversight in issue building the article

Nielbock et al.: The Herschel PACS photometer calibration. Exp. Astron. 36, 631660 (2013). doi:10.1007/s10686-013-9348-z

was published in an earlier issue instead of being included in this "Topical Issue on Calibration of the Herschel Space Observatory".

The publisher wishes to apologize for this mistake.

The online version of the original article can be found at http://dx.doi.org/10.1007/s10686-013-9348-z.

M. Nielbock $(\bowtie) \cdot$ Z. Balog · U. Klaas · H. Linz

Max-Planck-Institut für Astronomie, Königstuhl 17, 69117 Heidelberg, Germany

e-mail: nielbock@mpia.de

B. Altieri · M. Sánchez-Portal

European Space Astronomy Centre (ESAC)/ESA, Villanueva de la Cañada, 28691 Madrid, Spain

N. Billot

Instituto de Radio Astronomía Milimétrica, Avenida Divina Pastora,

7, Local 20, 18012 Granada, Spain

T. Müller

Max-Planck-Institut für extraterrestrische Physik, Gießenbachstraße,

85741 Garching, Germany

K. Okumura · M. Sauvage

Laboratoire AIM, CEA, Université Paris Diderot, IRFU/Service d'Astrophysique,

Bat. 709, 91191 Gif-sur-Yvette, France 